\title{
Differential Pulse-Width Pair Brillouin Optical Time-Domain Analysis Employing Raman Amplification and Optical Pulse Coding
}

\author{
M. Taki, M. Soto, F. Di Pasquale, and G. Bolognini
}

\section{Introduction}

One of the most adopted distributed sensing techniques exploits Brillouin optical time-domain analysis (BOTDA) due to its capability of providing an accurate simultaneous measurement of strain and temperature. The possibility of this technique, based on stimulated Brillouin scattering (SBS), to achieve long sensing ranges with high spatial resolution using standard single-mode fiber (SMF) enables many practical applications in structural health monitoring, where spatial resolution values ranging in the $\mathrm{cm}$ scale are in some cases required over long distances (e.g., crack detection). However, the spatial resolution in standard BOTDA systems is limited to $1 \mathrm{~m}$ due to the acoustic-phonon lifetime ( 10 ns), inducing a broadening of the Brillouin gain spectrum when pulse widths shorter than $10 \mathrm{~ns}$ are used, thus leading to a reduction of the Brillouin peak gain [1] and to inaccuracies in Brillouin frequency shift (BFS) measurements. In order to achieve spatial resolutions smaller than $1 \mathrm{~m}$, one promising technique is given by BOTDA employing differential pulse-width pair (DPP-BOTDA), which has been successfully employed for submeter spatial resolution [1-3], although limited in sensing range due to poor signal to noise (SNR) induced in the DPP subtraction process. Optical pulse coding has been applied to DPP-BOTDA [1, 2] overcoming the SNR limitations, in order to achieve longer distances. In this paper we employ bidirectional Raman amplification together with optical pulse coding to DPP-BOTDA, providing distributed sensing over long ranges together with submeter spatial resolution.

M. Taki $\bullet$ M. Soto $\bullet$ F. Di Pasquale

TeCIP Institute, Scuola Superiore Sant'Anna, Pisa, Italy

G. Bolognini $(\square)$

IMM Institute, Consiglio Nazionale delle Ricerche, Bologna, Italy

e-mail: bolognini@bo.imm.cnr.it 


\section{Experimental Setup and Results}

The experimental setup is shown in Fig. 1. The output of a distributed feedback (DFB) laser is split into two branches. In the probe (CW signal) branch, two sideband components are generated (suppressed carrier technique). In the pump (pulsed beam) branch, an erbium-doped fiber amplifier (EDFA) and a Mach-Zehnder modulator (MZM) driven by a waveform generator are used to amplify the DFB laser output and then to generate sequences of Simplex optical codes (127 bit) [4].

Bidirectional distributed Raman amplification [5] has been implemented by coupling two Raman pumps at 1,450 nm (in forward and backward directions) into a 93 km single-mode fiber (SMF); we employed a depolarized fiber Raman laser (FRL) as forward-propagating pump and a low-RIN depolarized pump based on polarization-multiplexed Fabry-Perot (FP) lasers as backward-propagating pump. We designed the optimal input power levels for Raman pumps and signals employing an in-house developed numerical method [6] based on the solution of a coupled differential equation system. At the receiver, a circulator and a fiber Bragg grating filter have been used, together with a linear-gain EDFA preamplifying the received traces.

The position of another EDFA before the MZM for the Brillouin pump branch avoids unwanted distortions in the coded light stream. Automated feedback controls for both signal and pump MZMs ensure time stability and improved measurement repeatability. A polarization scrambler is used in the signal branch to provide signal light depolarization and to reduce polarization-dependent gain occurring in SBS.

Finally, a $400 \mathrm{MHz}$ PIN photo-receiver and an analogue-to-digital converter (ADC) have been connected to a computer for trace acquisition. A differential pulsewidth pair of $60 / 56 \mathrm{~ns}$ has been used, corresponding to a theoretical limit of spatial resolution equal to $40 \mathrm{~cm}$.

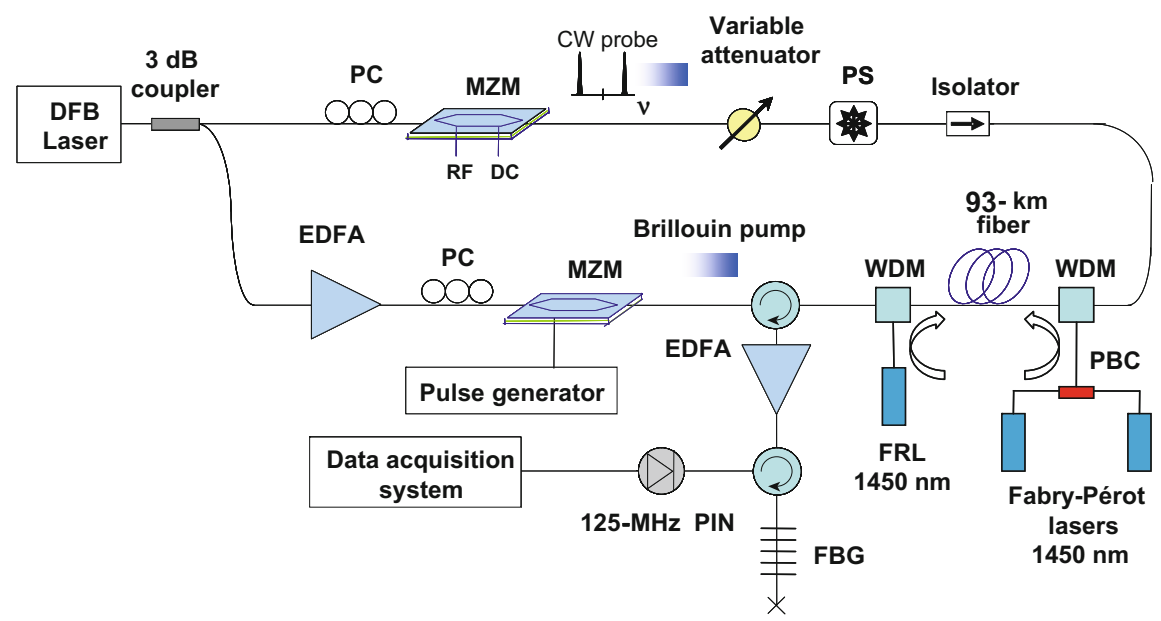

Fig. 1 Experimental setup 


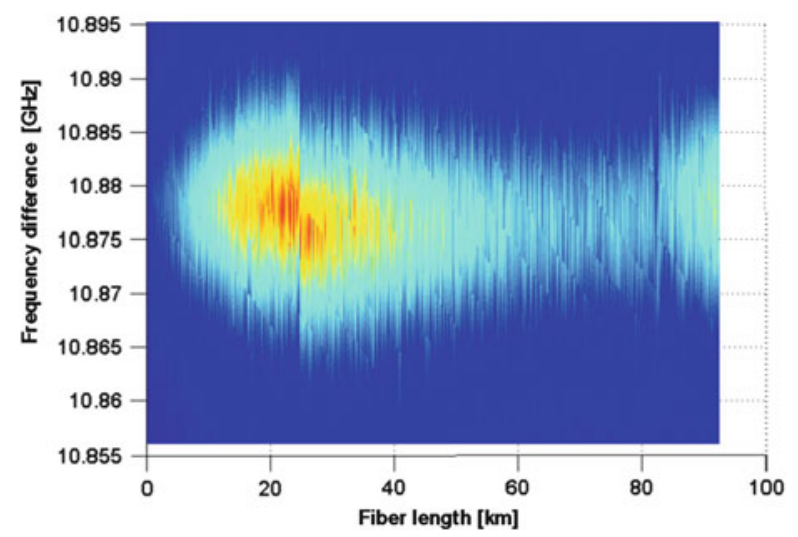

Fig. 2 Contour plot of Brillouin gain spectrum versus fiber length

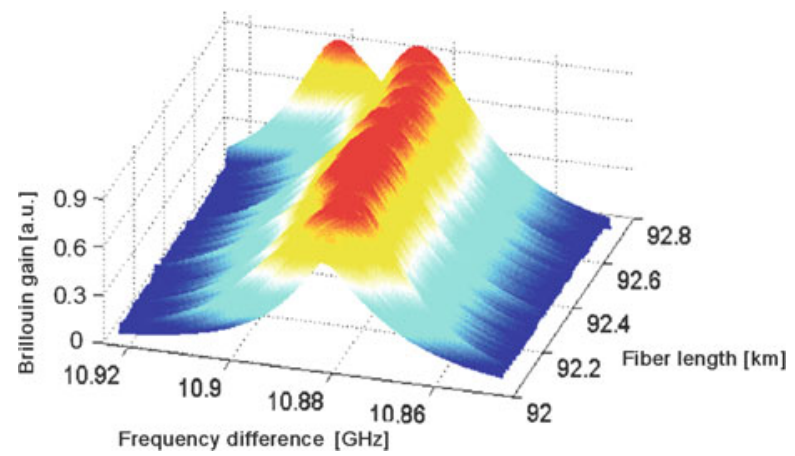

Fig. 3 Frequency spectrum of Brillouin gain versus fiber length

In order to acquire the whole Brillouin gain spectrum (BGS) through the fiber length, the $\mathrm{CW}$ probe frequency is tuned through an RF signal generator. For each frequency value, the coded traces for each pulse-width value are then decoded and the trace pairs are then subtracted as required in DPP scheme. The contour plot of BGS (top view) along the fiber length after DPP subtraction is shown in Fig. 2.

Considering the used power values and analyzing the spectral shape of the Brillouin gain throughout the fiber length, pump depletion effects can be considered as negligible. To verify the sensor performance, $10 \mathrm{~m}$ of fiber at $\sim 92.7 \mathrm{~km}$ distance have been placed inside a temperature-controlled chamber (TCC) heated to $43{ }^{\circ} \mathrm{C}$.

The BGS versus distance is shown in Fig. 3, showing the clear change in BGS peak near fiber end as a consequence to fiber heating. Temperature (or strain) estimations are carried out employing the Brillouin frequency shift (BFS) parameter, i.e., the frequency peak of measured BGS, which exhibits a linear behavior with temperature (or strain) variations [1]. For our experiment, Fig, 4 reports the BFS near the fiber end $(92.7 \mathrm{~km})$, also pointing out the attained spatial resolution in 


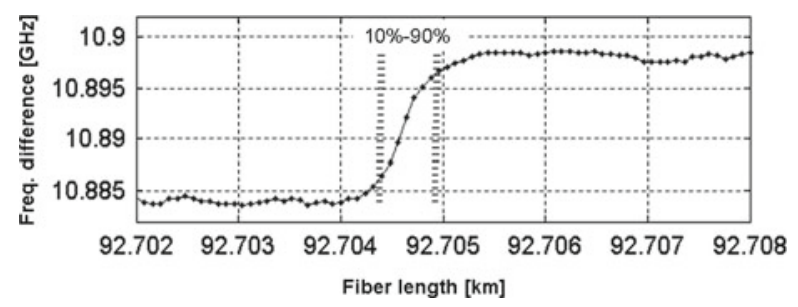

Fig. 4 Brillouin frequency shift versus fiber length (around $93 \mathrm{~km}$ )

correspondence to a temperature step, resulting to be $\sim 50 \mathrm{~cm}$ (10-90\% response), pretty close to the theoretical limit of $40 \mathrm{~cm}$. The temperature-strain resolution along the fiber length can be calculated from the standard deviation in BFS; in our setup the worst-temperature (strain) resolution has been estimated to be $\sim 1.7^{\circ} \mathrm{C}$ $(\sim 34 \mu \varepsilon)$.

As a conclusion, combining the use of a return-to-zero (RZ)-format Simplex coding together with optimized bidirectional Raman amplification allows for efficient distributed sensing based on Brillouin optical time-domain analysis (BOTDA) employing differential pulse-width pair (DPP) technique; in a long-distance experiment, we showed distributed measurements over $\sim 93 \mathrm{~km}$ of SMF, achieving temperature (strain) resolution better than $1.7{ }^{\circ} \mathrm{C}(34 \mu \varepsilon)$ and a spatial resolution better than $50 \mathrm{~cm}$ throughout the fiber length.

\section{References}

1. Liang, H., Li, W., Linze, N., Chen, L., and Bao, X., "High-resolution DPP-BOTDA over $50 \mathrm{~km}$ LEAF using return-to-zero coded pulses," Opt. Lett. 35(10), 1503-1505, (2010).

2. M. A. Soto, M. Taki, G. Bolognini, F. Di Pasquale, "Optimization of a DPP-BOTDA sensor with $25 \mathrm{~cm}$ spatial resolution over $60 \mathrm{~km}$ standard single-mode fiber using Simplex codes and optical pre-amplification,” Opt. Exp. 20(7), 6860-6869 (2012).

3. Y. Dong, X. Bao, W. Li, "Differential Brillouin gain for improving the temperature accuracy and spatial resolution in a long-distance distributed fiber sensor," Applied Opt., 48(22), 4297-4301 (2009).

4. M. D. Jones, "Using Simplex Codes to Improve OTDR Sensitivity", IEEE Photon. Technol. Lett., 15(7), 822-824 (1993).

5. S. Faralli, G. Bolognini, G. Sacchi, S. Sugliani, F. D. Pasquale, "Bidirectional higher order cascaded Raman amplification benefits for 10-Gb/s WDM unrepeated transmission systems," J. Lightwave Technol. 23(8), 2427-2433 (2005).

6. G. Bolognini, F. Di Pasquale, "Transient effects in gain clamped discrete Raman amplifiers", IEEE Photon. Technol. Lett., 16(1), 66-68 (2004). 